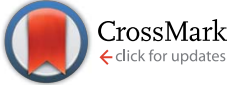

Cite this: Chem. Sci., 2016, 7, 6463

\title{
Interaction of methanol with the oxygen-evolving complex: atomistic models, channel identification, species dependence, and mechanistic implications $\uparrow$
}

\begin{abstract}
Marius Retegan $\ddagger$ and Dimitrios A. Pantazis*
Methanol has long being used as a substrate analogue to probe access pathways and investigate water delivery at the oxygen-evolving complex (OEC) of photosystem-II. In this contribution we study the interaction of methanol with the OEC by assembling available spectroscopic data into a quantum mechanical treatment that takes into account the local channel architecture of the active site. The effect on the magnetic energy levels of the $\mathrm{Mn}_{4} \mathrm{Ca}$ cluster in the $\mathrm{S}_{2}$ state of the catalytic cycle can be explained equally well by two models that involve either methanol binding to the calcium ion of the cluster, or a second-sphere interaction in the vicinity of the "dangler" Mn4 ion. However, consideration of the latest ${ }^{13} \mathrm{C}$ hyperfine interaction data shows that only one model is fully consistent with experiment. In contrast to previous hypotheses, methanol is not a direct ligand to the OEC, but is situated at the end-point of a water channel associated with the $\mathrm{O} 4$ bridge. Its effect on magnetic properties of plant PS-II results from disruption of hydrogen bonding between $\mathrm{O} 4$ and proximal channel water molecules, thus enhancing superexchange (antiferromagnetic coupling) between the Mn3 and Mn4 ions. The same interaction mode applies to the dark-stable $S_{1}$ state and possibly to all other states of the complex. Comparison of protein sequences from cyanobacteria and plants reveals a channel-altering substitution (D1-Asn87 versus D1-Ala87) in the proximity of the methanol binding pocket, explaining the speciesdependence of the methanol effect. The water channel established as the methanol access pathway is the same that delivers ammonia to the Mn4 ion, supporting the notion that this is the only directly solvent-accessible manganese site of the OEC. The results support the pivot mechanism for water binding at a component of the $S_{3}$ state and would be consistent with partial inhibition of water delivery by methanol. Mechanistic implications for enzymatic regulation and catalytic progression are discussed.
\end{abstract}

Received 26th May 2016 Accepted 28th June 2016

DOI: $10.1039 /$ c6sc02340a

www.rsc.org/chemicalscience

\section{Introduction}

A fundamental aspect of mechanistic regulation in metalloenzymes is how the active site, where catalytic transformation of substrates occurs, is connected to the protein environment. Connections may include solvent-permeable channels for the transport of substrates and products, pathways for the uptake or release of protons, and-in the case of redox transformationselectron transfer pathways to other sites of the enzyme or to peripheral cofactors. Such channels and pathways are not always easily identifiable from crystallographic models, and when they are (for example, in the form of ordered water chains) their function with respect to catalytic activity is not necessarily obvious.

Max Planck Institute for Chemical Energy Conversion, Stiftstrasse 34-36, 45470 Mülheim an der Ruhr, Germany.E-mail: dimitrios.pantazis@cec.mpg.de

$\dagger$ Electronic supplementary information (ESI) available: Additional geometric and energetic data, sequence alignments, and Cartesian coordinates of all models. See DOI: $10.1039 / \mathrm{c} 6 \mathrm{sc} 02340 \mathrm{a}$

\$ Present address: European Synchrotron Radiation Facility, 71 avenue des Martyrs, 38000 Grenoble, France.
The water-oxidizing tetramanganese-calcium cluster of photosystem-II (PS-II) that forms the inorganic core of the oxygen-evolving complex (OEC, Fig. 1) 1-6 $^{1-6}$ is a prime example of a metallocofactor embedded in a membrane enzyme of such complexity $^{7-10}$ that many structural aspects of substrate delivery, product evolution, and proton removal remain insufficiently defined. Driven by photo-induced charge separation at the chlorophyll complex $\mathrm{P} 60^{+}$, the OEC cycles through five redox states denoted $\mathrm{S}_{i}$, where $i$ indicates the number of accumulated oxidizing equivalents $(i=0-4) \cdot{ }^{\mathbf{1 1}, 12}$ Stored in the dark the catalytic centers adopt predominantly the $S_{1}$ state, whereas the $S_{0}, S_{2}$ and $\mathrm{S}_{3}$ states are metastable. In these intermediates the $\mathrm{Mn}$ oxidation states evolve from $\mathrm{Mn}$ (III) ${ }_{3} \mathrm{Mn}$ (IV) in $\mathrm{S}_{0}$ to $\mathrm{Mn}(\mathrm{IV})_{4}$ in $\mathrm{S}_{3}$ (for a recent review on oxidation state assignments, see Krewald et $\left.a .^{13}\right)$. O-O bond formation ${ }^{\mathbf{1 4 , 1 5}}$ occurs upon advancement to the reactive and still unobserved $\mathrm{S}_{4}$ state; following $\mathrm{O}_{2}$ release the cluster is reset to the most reduced $\mathrm{S}_{0}$ state. Strict control of accessibility and ordered substrate binding at the active site, buried into the membrane-embedded part of PS-II, have long been recognized to be functionally critical in regulating the complex chemistry of water oxidation and avoiding side 


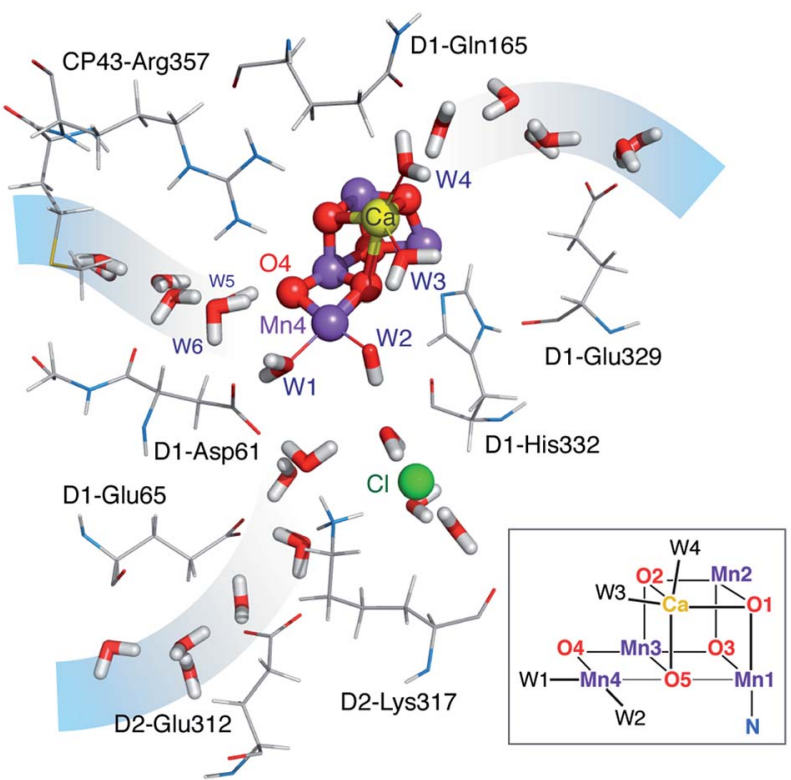

Fig. 1 The OEC and the proximal branches of associated water channels. Selected residues are shown for orientation. Coordinates are taken from QM/MM models of the $S_{2}$ state. ${ }^{17}$ Inset: schematic diagram and labelling of the inorganic core.

reactions. ${ }^{16}$ A detailed understanding of the water oxidation process therefore requires a positive identification of how, when, and where precisely the substrate water binds at the cluster-a great challenge that requires the substrate to be differentiated from other water-derived ligands, solvent or structural water, and water involved in proton relays.

Based on analysis of crystallographic models and molecular dynamics simulations, ${ }^{\mathbf{1 8 - 3 0}}$ there is presently consensus on the existence of at least three well-defined water channels associated with the $\mathrm{Mn}_{4} \mathrm{CaO}_{5}$ cluster (Fig. 1). ${ }^{31}$ Two of them appear to make contact with the Mn4 ion or its immediate environment, while the third one with the calcium ion, although the presence of water molecules bridging between the two Mn4-bound waterderived terminal ligands and the two $\mathrm{Ca}^{2+}$-bound waters complicates the assignment of distinct contact points. The role of these channels in water delivery, proton removal, ${ }^{32}$ and dioxygen release remains undefined and contested, ${ }^{19-21,24,30,33-35}$ and so is the structural basis of these functionalities.

Substrate analogues such as ammonia and methanol can interact with the OEC or at least approach the cluster sufficiently to serve as spectroscopic probes. ${ }^{36-66}$ By perturbing the geometric and electronic structure of the manganese cluster, these molecules alter the spectroscopic signature of the complex compared to the native system. Direct structural interpretations of the observed effects are usually unattainable. However, when such data are combined with spectroscopy-oriented quantum chemical studies ${ }^{\mathbf{4 4 , 6 7 - 7 4}}$ it is often possible to arrive at experimentally consistent atomistic models that reveal the mode of interaction and the role of specific channels, providing insight into the local enzymatic architecture as well as on regulatory and mechanistic aspects of water oxidation.
Methanol is a key molecule in this respect, because it affects the electronic structure and modifies the EPR signatures ${ }^{75}$ of all states. ${ }^{4-65}$ A major effect is that it increases the energy separation between the lowest magnetic levels of the OEC, ${ }^{\mathbf{4 8 , 5 1 , 6 0 , 6 2}}$ stabilizing the $S=1 / 2$ ground spin states of the $S_{0}$ and $S_{2}$ states and the diamagnetic ground state of $S_{1}$. Other effects relate to the amplitude enhancement of specific spectral forms, for example of the $g \approx 2$ multiline signal of the $S_{2}$ state in plants over the $g \geq 4$ component. It is not clear whether a common interaction mode is valid for all catalytic states. Interestingly, higher plants and cyanobacteria behave differently to addition of methanol, ${ }^{62,76,77}$ the latter appearing less sensitive in its spectroscopic response than the former, but the origin of this species dependence is not understood. Efforts to identify possible methanol binding modes employed deuterated methanol $\left(\mathrm{CD}_{3} \mathrm{OH}\right)$ in electron spin-echo envelope modulation (ESEEM) experiments for the $\mathrm{S}_{2}$ and $\mathrm{S}_{0}$ states. ${ }^{49,59,78}$ For the $\mathrm{S}_{2}$-state studies ${ }^{49,59}$ the inferred $\mathrm{Mn}-{ }^{2} \mathrm{H}$ distances were interpreted as consistent with direct ligation of methanol to a $\mathrm{Mn}$ center, but the two ESEEM data sets and their interpretation in the context of a structural model of the OEC differ substantially, being consistent either with methanol displacing a waterderived ligand bound to Mn4, or with methanol displacing Glu189, an amino acid residue directly coordinated to Mn1.62 The concept of direct ligation to the Mn1 and Mn4 sites with different affinities was recently used also in the interpretation of D1-Tyr161 radical "split signals" that can be generated from the $\mathrm{S}_{2}$ state under different illumination conditions. ${ }^{63}$ On the other hand, the ESEEM study of the $S_{0}$ state did not result in observation of specific interactions, disfavoring direct methanol binding to the OEC. ${ }^{78}$ It is clear that neither the data can be reconciled, nor any of the interpretations supported with confidence. Beyond the intrinsic limitations in the treatment of deuteron dipolar couplings, the involvement of Mn1 as a potential binding site ${ }^{\mathbf{4 9 , 5 9 , 6 3}}$ conflicts with channel accessibility studies, ${ }^{20,25}$ while explanations that invoke drastic reorganizations of first-sphere ligands seem incompatible with the small observed spectroscopic perturbations and the retention of catalytic activity. Similar ambiguities pertain to structural interpretations of methanol effects on the miss parameter for S-state advancement. ${ }^{\mathbf{6 1}}$

A significant advance in this line of study was achieved recently by Oyala et al. ${ }^{65}$ who used ${ }^{13} \mathrm{C}$-labeled methanol in pulse EPR studies of spinach PS-II poised in the $\mathrm{S}_{2}$ state. This is the most direct study of methanol interaction with the OEC to date. The measured ${ }^{13} \mathrm{C}$ hyperfine interactions, compared with a reference $\mathrm{Mn}(\mathrm{III}, \mathrm{IV})$ complex, argued very strongly against direct binding of methanol to a $\mathrm{Mn}$ ion. Maps of the ${ }^{13} \mathrm{C}$ dipolar hyperfine couplings identified two plausible regions for methanol interaction, either in the cluster of water molecules surrounding $\mathrm{Ca}^{2+}$, possibly by directly replacing one of the calcium-bound waters, or in the vicinity of Mn4 where water molecules can form hydrogen bonds with oxygen bridges, or a combination of the above. Two of the channels shown in Fig. 1 may therefore be implicated in methanol delivery, but no further differentiation can be made and no unique structural models can be proposed. This leaves still open the crucial 
question of substrate delivery and identification; for example, if $\mathrm{MeOH}$ indeed replaces the Ca-bound $\mathrm{W} 3$ and remains bound in later stages of the catalytic cycle, this would likely exclude the possibility of a calcium-bound water acting as a substrate in a nucleophilic attack scenario for $\mathrm{O}-\mathrm{O}$ bond formation. ${ }^{79-82}$

In this study we use available experimental data, including the valuable dataset on ${ }^{13} \mathrm{C}$ hyperfine interactions, ${ }^{65}$ to evaluate a series of quantum chemical models that include explicit binding of $\mathrm{MeOH}$ at different sites in either protonated or deprotonated form, as well as many second-sphere interaction modes. Based on the magnetic and spectroscopic properties of these models, we conclude that the experimental observations have a unique structural interpretation, identifying a secondsphere interaction site for $\mathrm{MeOH}$ along a water channel associated with the $\mathrm{O} 4$ bridge. The atomistic details of the model provide a novel explanation of how the energy level splittings are affected by modulation of the Mn3-O4-Mn4 superexchange pathway in the $\mathrm{S}_{2}$ and potentially in other $\mathrm{S}$ states. Comparison of PS-II amino acid sequences from plants and cyanobacteria reveal a conserved difference close to the proposed site of interaction, offering a structure-based explanation for the species-dependent response to methanol. By combining the present results with recent studies of ammonia interaction with the $\mathrm{OEC}^{\mathbf{4 4 , 6 4 , 6 6 , 8 3}}$ it is possible to assign a unique channel as active in delivery of substrate analogues to the OEC. Taking into account the studies of the $\mathrm{S}_{2}-\mathrm{S}_{3}$ transition and the associated "pivot" mechanism for water binding, ${ }^{73}$ we conclude that the dangler manganese (Mn4) is the only directly accessible $\mathrm{Mn}$ ion of the cluster and that substrate inclusion to the OEC occurs through initial binding at this site.

\section{Methodology}

\section{Generation of structural models}

Our approach for creating atomistic models to describe the interaction of methanol with the OEC is based on systematically substituting first and second sphere water molecules by $\mathrm{MeOH}$ and exploring the conformational space for each substitution. The "open cubane" form of the cluster that corresponds to the multiline $S=1 / 2$ signal of the $\mathrm{S}_{2}$ state $^{70}$ was used as starting point. We employed our previously described QM/MM models of the $S_{2}$ state $^{17}$ for preliminary screening and set the size of the final QM-only models to sufficiently include all first-sphere hydrogen bonding interactions. The final models consist of 240-242 atoms. The formally "open" coordination site of the five-coordinate $\mathrm{Mn}$ (III) ion $\mathrm{Mn} 1$ was also considered as a possible interaction site. However, after multiple attempts we conclude that this site is both inaccessible, in line with previous studies of channel architecture, ${ }^{20}$ and unsuitable for methanol binding as it lies along the Jahn-Teller axis of the Mn(III) ion.

Twelve water molecule positions were considered as candidates for $\mathrm{MeOH}$ substitution: four are direct $\mathrm{Mn}$ or Ca ligands (W1-W4) and eight are second-sphere crystallographically characterized water molecules (W5-W12) that participate in hydrogen-bonding interactions with first-sphere residues. ${ }^{7,8}$ The W1 and $\mathrm{W} 2$ ligands to $\mathrm{Mn} 4$ were considered as $\mathrm{H}_{2} \mathrm{O}$ and $\mathrm{OH}{ }^{69,72}$ The labeling used for the water molecules is shown in Fig. 2.
The correspondence of these labels with the identification numbers of the most recent crystallographic models with PDB codes 3ARC, 3WU2, and 4UB6 is provided in Table S1 of the ESI. $\dagger$ For each of the twelve positions, after replacing the $\mathrm{H}_{2} \mathrm{O}$ for $\mathrm{MeOH}$ several conformations and protonation isomers were generated either by automatically scanning dihedral angles or by manually reorienting specific groups. Of the several tens of conformers thus generated and geometry-optimized, eventually we selected twenty "best-case" models that we consider representative of all conceivable $\mathrm{MeOH}$ arrangements. Given that $\mathrm{MeOH}$ is only mildly perturbing the spectroscopic signature of the OEC without significantly altering its electronic structure, any models in which the initial positioning of $\mathrm{MeOH}$ resulted in extensive structural rearrangements upon optimization such as decoordination of first-sphere residues with concomitant large changes in electronic structure, were discarded.

Geometries were optimized with the ORCA program package ${ }^{84}$ using the BP86 functional ${ }^{85,86}$ combined with the D3 model for dispersion corrections, ${ }^{87}$ the COSMO model ${ }^{88}$ with a dielectric of 8 to simulate the effect of the protein environment, and the zeroth-order regular approximation (ZORA) Hamiltonian ${ }^{89-91}$ for scalar relativistic effects. ZORA-recontracted $^{92}$ Karlsruhe basis sets of polarized valence triple- $\zeta(\mathrm{Mn}$, $\mathrm{Ca}, \mathrm{O}, \mathrm{N})$ and double- $\zeta(\mathrm{C}, \mathrm{H})$ quality were used, ${ }^{93,94}$ with increased integration grids (Grid6 and IntAcc 6.0 in ORCA notation) and tight SCF convergence criteria. Fully decontracted def2-TZVP/J basis sets ${ }^{95}$ were employed in density fitting.

\section{Spin states and magnetic properties}

For each model a complete set of broken-symmetry (BS) DFT calculations was carried out in terms of single and double spin flips to compute the six pairwise exchange coupling constants $J_{i j}$ that parameterize the magnetic coupling between the four $\mathrm{Mn}$ ions in an Ising-type Hamiltonian. These calculations employed the hybrid meta-GGA functional TPSSh, ${ }^{96}$ which has been

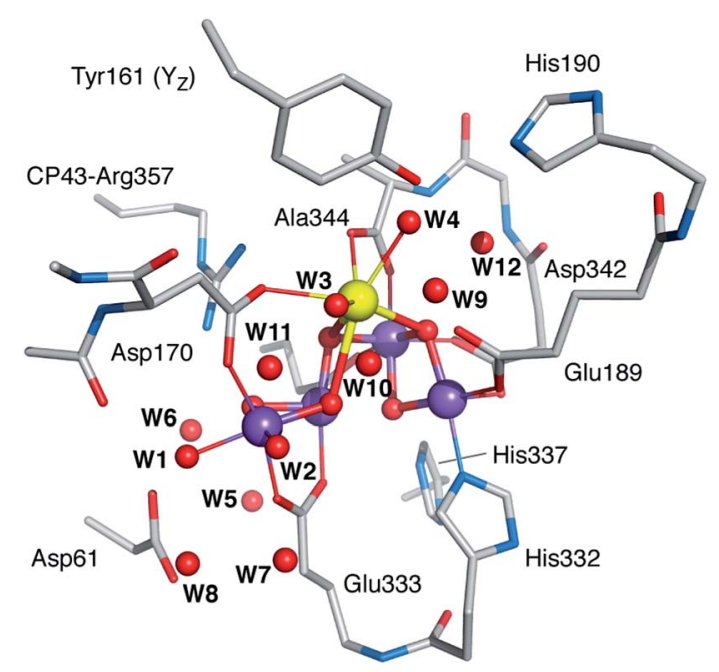

Fig. 2 Computational model of the OEC used in the present work, with labelling of the relevant water molecules (hydrogen atoms omitted for clarity). 
shown to perform extremely well in the prediction of magnetic properties for high-valent Mn clusters. ${ }^{67,72,97-99}$ These BS solutions are not spin eigenfunctions and it is meaningless to attempt any direct comparison with experiment, either with respect to an observed ground spin state or with respect to measured energy differences between magnetic sublevels. It is unfortunate that this crucial point is missed in part of the literature. The exchange coupling constants obtained from the BS solutions serve to form and diagonalize the HeisenbergDirac-van Vleck (HDvV) Hamiltonian

$$
\hat{H}_{\mathrm{HDvV}}=-2 \sum_{i<j} J_{i j} \hat{S}_{i} \hat{S}_{j}
$$

thereby obtaining the complete spectrum of spin eigenstates (e.g. 320 magnetic levels for the $\mathrm{S}_{2}$ state including spin multiplets) and their relative energies. These results constitute a valid basis for comparisons with experiment; we use the spin states and energy differences between HDvV states thus identified to evaluate all models.

\section{Spectroscopic properties}

The hyperfine coupling results from the interaction of the spin of the unpaired electron(s) $S$ and the magnetic nuclear moments $I$ :

$$
\hat{H}=\hat{S} \mathbf{A}^{(\mathrm{A})} \hat{I}^{(\mathrm{A})}=\sum_{k=x, y, z} \sum_{l=x, y, z} A_{k l}^{(\mathrm{A})} S_{k} I_{l}^{(\mathrm{A})}
$$

The tensor parameterizing the interaction, $\mathbf{A}^{(\mathrm{A})}$, consists of three contributions: the Fermi contact, the spin dipolar, and the spin-orbit coupling contribution:

$$
A_{k l}^{(\mathrm{A})}=A_{k l}^{(\mathrm{A} ; \mathrm{FC})}+A_{k l}^{(\mathrm{A} ; \mathrm{SD})}+A_{k l}^{(\mathrm{A} ; \mathrm{SO})}
$$

In the framework of density functional methods analytical solutions for the three terms have been derived ${ }^{\mathbf{1 0 0}}$ according to the following equations:

$$
\begin{gathered}
A_{k l}^{(\mathrm{A} ; \mathrm{FC})}=\delta_{k l} \frac{8 \pi}{3} \frac{g_{\mathrm{e}} g_{\mathrm{N}}^{(\mathrm{A})} \beta_{\mathrm{e}} \beta_{\mathrm{N}}}{2 S} \sum_{\mu \nu} P_{\mu \nu}^{\alpha-\beta}\left\langle\varphi_{\mu}\left|\delta\left(\mathbf{r}_{\mathrm{iA}}\right)\right| \varphi_{\nu}\right\rangle \\
A_{k l}^{(\mathrm{A} ; \mathrm{SD})}=\frac{g_{\mathrm{e}} g_{\mathrm{N}}^{(\mathrm{A})} \beta_{\mathrm{e}} \beta_{\mathrm{N}}}{2 S} \sum_{\mu \nu} P_{\mu \nu}^{\alpha-\beta}\left\langle\varphi_{\mu}\left|r_{\mathrm{iA}}^{-5}\left(r_{\mathrm{iA}}^{2} \delta_{k l}-3 r_{\mathrm{iA} ; k} r_{\mathrm{iA} ; l}\right)\right| \varphi_{\nu}\right\rangle \\
A_{k l}^{(\mathrm{A} ; \mathrm{SO})}=-\frac{g_{\mathrm{e}} g_{\mathrm{N}}^{(\mathrm{A})} \beta_{\mathrm{e}} \beta_{\mathrm{N}}}{2 S} \sum_{\mu \nu} \frac{\partial P_{\mu \nu}^{\alpha-\beta}}{\partial \hat{I}_{k}^{(\mathrm{A})}} P_{\mu \nu}^{\alpha-\beta}\left\langle\varphi_{\mu}\left|\hat{z}_{l}^{\mathrm{SOMF}}\right| \varphi_{\nu}\right\rangle
\end{gathered}
$$

here, $g_{\mathrm{e}}$ is the $g$-value of the free electron, $g_{\mathrm{N}}^{(\mathrm{A})}$ is the $g$-value of nucleus $\mathrm{A}, \beta_{\mathrm{e}}$ is the Bohr magneton, $\beta_{\mathrm{N}}$ is the nuclear magneton, $P^{\alpha-\beta}$ is the spin-density matrix, $I^{(\mathrm{A})}$ is the nuclear spin operator of nucleus $A, z^{\mathrm{SOMF}}$ is the spin-orbit coupling operator, and

$$
r_{\mathrm{iA}}=\left|\mathbf{r}_{\mathrm{iA}}\right|=\left|\mathbf{r}_{\mathrm{i}}-\mathbf{R}_{\mathrm{A}}\right|
$$

where $r_{\mathrm{i}}$ is the position vector of electron $\mathrm{i}$ and $\mathbf{R}_{\mathrm{A}}$ is the position vector of nucleus $A$. The summation runs over the basis functions $\varphi$. The first two terms are expectation values over the spin density; the isotropic Fermi contact term arises from the spin density on the nucleus under investigation, while the spin dipolar part is due to the through-space interaction between the electron and nuclear spin magnetic moments. The third term is a second-order spin-orbit contribution, which is important mainly for heavier elements. In the present work, the ${ }^{13} \mathrm{C}$ hyperfine parameters were computed with the TPSSh functional combined with triple- $\zeta$ ZORA-recontracted basis sets on carbon, locally increased integration grids (SpecialGridIntAcc $=9.0$ ), and inclusion of relativistic picture-change effects. For each model we report the isotropic hyperfine value $\left(A_{\text {iso }}\right)$, defined as the sum of the Fermi contact term and the isotropic part of the spin-orbit coupling contribution, and the dipolar term $T$ defined as

$$
T=\frac{1}{2} A_{z z}^{(\mathrm{A} ; \mathrm{SD})}
$$

\section{Sequence alignment}

The sequences of the PS-II core proteins D1 (PsbA), D2 (PsbD), CP43 (PsbC) and CP47 (PsbB) from T. vulcanus, T. elongatus, and $S$. oleracea were aligned with ClustalW2. ${ }^{101,102}$

\section{Results and discussion}

\section{Atomistic models for methanol interaction}

Among the many structural models that were optimized and evaluated in terms of their properties, we have selected twenty representative structures (Fig. 3) that fully cover the range of possibilities with respect to methanol interaction with the OEC. The models are labeled according to the water molecule that is substituted by methanol, followed by an additional number that refers to the conformational variant. The models include structures where methanol is deprotonated when it binds as a first-sphere ligand; these are indicated with the letter " $\mathrm{d}$ " as a label suffix.

In all cases there is minimal structural perturbation of the inorganic core, even in models where methanol ligates directly to a metal ion of the OEC. The relative energies of these models, computed with three different approaches, are provided in Table $\mathrm{S} 2 . \dagger$ It is stressed however that comparison of these energies is only partially informative for four important reasons. First, a fundamental limitation is that not all models are isomers (e.g. W2-substituted models are unique, and a subset of models contain deprotonated methanol). Second, several models span a narrow energy range; this is particularly true for positions remote from the inorganic cluster that are not associated with large energetic perturbations (e.g. models W5-1, W7-1, and W10-1/2 are isoenergetic even though the access and interaction sites of methanol are fundamentally different). Third, for several structures the truncation of interactions that would propagate beyond the computational model employed in this study implies unknown uncertainties (for example, a high-energy 

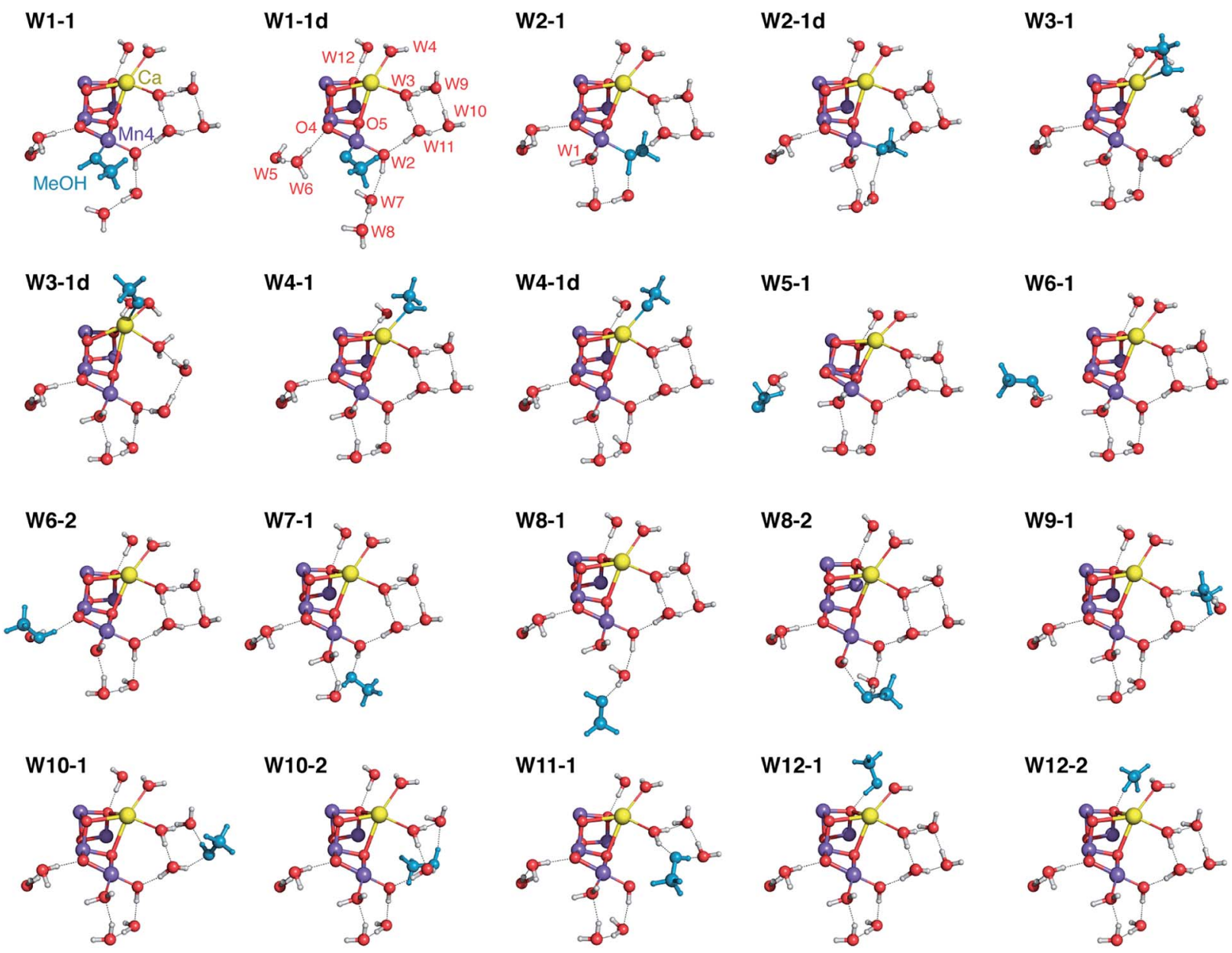

Fig. 3 Position of methanol (in blue) and of water molecules around the OEC cluster in selected optimized models. All protein residues are omitted for clarity.

model might be stabilized by hydrogen bonding or a lowenergy model might be destabilized by steric interactions with parts of the protein missing from the QM models). And fourth, relative energies are irrelevant when comparing models that involve different access channels. To appreciate this, let us assume that methanol in reality can only access the calcium site of the cluster; then it is of no relevance that W4-1 is 3-4 $\mathrm{kcal} \mathrm{mol}^{-1}$ higher in energy than W7-1 (Table S2 $\dagger$ ), because the latter would be impossible to exist. The only relevant energetic quantities in this case would be the relative permeation barriers through different channels of the enzyme, an aspect that is entirely missing from a cluster $\mathrm{QM}$ approach. Therefore, although relative energies are crucial in elucidating and comparing reaction pathways, ${ }^{14,15,103-107}$ they are of limited utility for the present study and make sense only when comparing closely related subsets, for example pairs of rotamers such as W6-1 and W6-2 (see Table S2 $\dagger$ ). A meaningful evaluation of models in the present case can be based only on comparison of the values of observable properties against experiment, so in the following we focus on the magnetism and spectroscopy of these twenty structures.

\section{Magnetic properties}

An important effect of methanol on the multiline signal of the $\mathrm{S}_{2}$ state is the increase of the energy gap $\Delta E$ between the ground $(S=1 / 2)$ and first excited $(S=3 / 2)$ states. ${ }^{62}$ For $T$. elongatus it is estimated that the native energy separation of $c a .13 \mathrm{~cm}^{-1}$ is increased to $c a .22 \mathrm{~cm}^{-1}$ upon addition of methanol, while for spinach the most recent study reported a change from $c a .3$ $\mathrm{cm}^{-1}$ to $c a .25 \mathrm{~cm}^{-1}{ }^{62}$ Older estimates in the literature place the native $\Delta E$ of spinach to $c a .6 \mathrm{~cm}^{-1},{ }^{47}$ while for methanol-treated samples previous estimates included $12 \mathrm{~cm}^{-1}, 5030 \mathrm{~cm}^{-1,},{ }^{47}$ and $36.5 \mathrm{~cm}^{-1}{ }^{46}$ Therefore, our principal target here is to identify methanol interaction sites that correlate with an increase of $\Delta E$.

Table 1 lists all exchange coupling constants for all models of the present study. The spin of the ground and first excited states as well as their energy difference $\Delta E$, obtained from diagonalization of the HDvV Hamiltonian, are also reported in Table 1 for each model. The results computed for the "native" $S_{2}$ state model of the OEC are provided as reference. Before we proceed, it is important to clarify a methodological point: given that the present model of the unperturbed, "native" state of the OEC does not extend too far from the inorganic cluster, it cannot capture differences between species that originate in protein 
Table 1 Exchange coupling constants $J\left(\mathrm{~cm}^{-1}\right)$, ground spin state $\left(\mathrm{S}_{\mathrm{GS}}\right)$, first excited spin state $\left(\mathrm{S}_{\mathrm{ES}}\right)$, their energy separation $\Delta E_{\mathrm{GS}-\mathrm{ES}}\left(\mathrm{cm}{ }^{-1}\right)$, and the change $\Delta \Delta E_{\mathrm{GS}-\mathrm{ES}}(\%)$ with respect to the model of the native system

\begin{tabular}{|c|c|c|c|c|c|c|c|c|c|c|}
\hline & $J_{12}$ & $J_{13}$ & $J_{14}$ & $J_{23}$ & $J_{24}$ & $J_{34}$ & $\mathrm{~S}_{\mathrm{GS}}$ & $\mathrm{S}_{\mathrm{ES}}$ & $\Delta E_{\mathrm{GS}-\mathrm{ES}}$ & $\Delta \Delta E_{\mathrm{GS}-\mathrm{ES}}$ \\
\hline Native & -16.1 & 2.6 & 1.4 & 23.7 & 2.0 & -13.3 & $1 / 2$ & $3 / 2$ & 21.0 & \\
\hline W1-1 & -15.9 & 3.0 & 1.1 & 23.5 & 2.0 & -13.5 & $1 / 2$ & $3 / 2$ & 20.8 & -1 \\
\hline W1-1d & -16.4 & 6.4 & -0.4 & 24.5 & 2.1 & -12.4 & $1 / 2$ & $3 / 2$ & 17.3 & -18 \\
\hline W2-1 & -14.4 & 1.1 & 1.9 & 23.7 & 1.7 & -14.7 & $1 / 2$ & $3 / 2$ & 22.0 & 5 \\
\hline W2-1d & -15.6 & 2.5 & 2.0 & 23.8 & 1.5 & -12.9 & $1 / 2$ & $3 / 2$ & 20.4 & -3 \\
\hline W3-1 & -15.7 & 2.8 & 1.6 & 23.1 & 2.1 & -13.9 & $1 / 2$ & $3 / 2$ & 21.0 & 0 \\
\hline W3-1d & -16.1 & 3.4 & 5.1 & 29.0 & 1.5 & -20.2 & $1 / 2$ & $3 / 2$ & 25.0 & 19 \\
\hline W4-1 & -17.1 & 2.8 & 1.6 & 20.2 & 1.8 & -11.6 & $1 / 2$ & $3 / 2$ & 20.2 & -4 \\
\hline W4-1d & -10.6 & 4.6 & 2.1 & 1.5 & 0.5 & -18.6 & $1 / 2$ & $3 / 2$ & 28.8 & 37 \\
\hline W5-1 & -17.2 & 3.2 & 1.1 & 18.5 & 1.8 & -15.6 & $1 / 2$ & $3 / 2$ & 24.4 & 16 \\
\hline W6-1 & -16.8 & 2.8 & 1.4 & 19.7 & 2.0 & -21.2 & $1 / 2$ & $3 / 2$ & 28.7 & 37 \\
\hline W6-2 & -15.8 & 4.7 & 0.5 & 28.5 & 1.4 & -8.6 & $1 / 2$ & $3 / 2$ & 14.3 & -32 \\
\hline W7-1 & -16.1 & 3.4 & 1.7 & 22.6 & 2.0 & -11.3 & $1 / 2$ & $3 / 2$ & 18.5 & -12 \\
\hline W8-1 & -16.2 & 2.6 & 1.3 & 24.3 & 1.9 & -13.9 & $1 / 2$ & $3 / 2$ & 21.6 & 3 \\
\hline W8-2 & -16.2 & 4.4 & 0.8 & 25.8 & 1.5 & -16.0 & $1 / 2$ & $3 / 2$ & 21.5 & 2 \\
\hline W9-1 & -14.6 & 1.7 & 1.9 & 24.7 & 1.9 & -11.2 & $1 / 2$ & $3 / 2$ & 18.5 & -12 \\
\hline W10-1 & -16.2 & 2.8 & 1.3 & 23.3 & 2.0 & -12.7 & $1 / 2$ & $3 / 2$ & 20.5 & -2 \\
\hline W10-2 & -15.3 & 2.6 & 1.8 & 23.5 & 2.1 & -12.8 & $1 / 2$ & $3 / 2$ & 19.9 & -5 \\
\hline W11-1 & -15.9 & 2.9 & 1.7 & 23.8 & 2.1 & -15.4 & $1 / 2$ & $3 / 2$ & 22.4 & 7 \\
\hline W12-1 & -16.2 & 2.8 & 1.2 & 22.3 & 2.0 & -12.6 & $1 / 2$ & $3 / 2$ & 20.4 & -3 \\
\hline W12-2 & -15.3 & 2.8 & 1.4 & 23.6 & 2.0 & -12.8 & $1 / 2$ & $3 / 2$ & 19.8 & -6 \\
\hline
\end{tabular}

sequence variations at sites that are not included in the model. The structural nature of these remote differences is in any case undefined, as no atomic-resolution structure of higher plant PS-II is still available. ${ }^{108}$ In this sense, the unperturbed $S_{2}$ state model used as the departure point in this study can be viewed in principle as "species-agnostic" and serves as a platform to study relative changes, i.e. the sign and magnitude of $\Delta \Delta E$ expected upon displacement of specific ligated or proximal waters by methanol.

In all cases the nature of the ground spin state $(S=1 / 2)$ and the first excited state $(S=3 / 2)$ remain the same, regardless of the type of methanol interaction. The exchange coupling constants and the energy splitting between the ground and the first excited states proved in general to be rather insensitive to water displacement by methanol, but there are also a few models where $\Delta E$ increases or decreases significantly. Focusing on the models that show a large increase as required for agreement with experiment, we can see that they belong to two groups: the first involves direct binding of methanol to calcium, but only in deprotonated (methoxy) form. These are the models W3-1d and W4-1d, the latter showing (together with W6-1) the largest increase in $\Delta E$ compared with the native system. The second group includes the two water sites that form part of the O4-related channel and is represented by models W5-1 and W6-1. Both groups correspond to two different channels, the calcium channel and the $\mathrm{O} 4$ channel of Fig. 1 respectively. By contrast, there is no water position associated with the third channel (W7, W8) that can reproduce to any extent the phenomenology when occupied by methanol. Direct ligation to Mn4 either has minimal effect on the predicted $\Delta E$, or it has the opposite effect than what is experimentally observed, when $\mathrm{MeOH}$ binds as methoxy in the W1 position (W1-1d). Therefore, from the perspective of the effect on magnetism, direct ligation to $\mathrm{Mn}$ is disfavored and the two models W4-1d and W6-1 stand out from the rest as most consistent with experiment, even though they represent fundamentally different binding modes.

The fact that W4-1d reproduces the large positive change in $\Delta E$ is somewhat unexpected because there is no direct interaction with a magnetic site. Nevertheless, the values in Table 1 show that coordination of $\mathrm{MeO}^{-}$to $\mathrm{Ca}^{2+}$ does affect the exchange coupling constants, particularly within the cuboidal $\mathrm{Mn}_{3} \mathrm{CaO}_{4}$ unit. The major cause for the change in $\Delta E$ is the diminished value of the ferromagnetic coupling constant $J_{23}$ compared to the native system. The changes in exchange coupling constants in this model as well as in W3-1d show that such alterations are not necessarily local and cannot always be properly analyzed as such. This serves as a warning that the interpretation of magnetic and spectroscopic responses under the assumption of locality of perturbations may sometimes be oversimplified.

The other model that emerged as a candidate for $\mathrm{MeOH}$ interaction is W6-1. Here the major change is observed for $J_{34}$. To understand how the presence and orientation of methanol at the W6 site regulates the magnetic coupling between Mn3 and Mn4, it is necessary to examine both W6-1, which shows a shift in $\Delta E$ that agrees best with experiment, and W6-2, which behaves in exactly the opposite way and shows the poorest agreement among all models in terms of $\Delta E$. In the native system W6 forms a hydrogen bond with $\mathrm{O} 4$. When methanol replaces W6 it can be oriented in a way that a hydrogen bond is also established between its hydroxy group and $\mathrm{O} 4$; this case is represented by W6-2. An additional hydrogen bonding interaction is established between W5 and MeOH. However, methanol can also be oriented in a way that abolishes hydrogen 
bonding with $\mathrm{O} 4$ and this is what happens in W6-1, where the $\mathrm{OH}$ group of methanol acts instead as a hydrogen bond donor to D1-Asp61 and as an acceptor from both W5 and CP43-Arg357 (Fig. 4). These differences have two implications. First, the orientation of methanol that is associated with a higher degree of hydrogen bonding, W6-1, is energetically favorable by $\mathrm{ca} .7$ kcal $\mathrm{mol}^{-1}$ compared to W6-2, because the positioning of methanol without hydrogen bonding to $\mathrm{O} 4$ but with three hydrogen-bonding interactions in total better stabilizes $\mathrm{MeOH}$ at the W6 site (Fig. 4, middle). Second, as expected ${ }^{99,109-112}$ the enhanced superexchange over $\mathrm{O} 4$ in the absence of the hydrogen bonding interaction compared to the native system leads to a more negative (antiferromagnetic) value for $J_{34}$, stabilization of the low-spin $S=1 / 2$ state of the OEC, and increase in $\Delta E$. The differences in exchange coupling constants mirror the trend in optimized Mn3-Mn4 distances, which are 2.753 $\AA$ for the "native" model, $2.735 \AA$ for W6-1, and $2.777 \AA$ for W6-2.

This atomistic description of the methanol effect on the magnetic structure of the cluster in the case of model W6-1 is almost entirely local compared to W4-1d. As such, it has obvious parallels with our previous qualitative description of the methanol effect on the $S_{2}$ state that was cast in terms of an effective interaction $J_{\text {eff }}$ between the terminal Mn4 and the $\mathrm{Mn}_{3} \mathrm{Ca}$ subunit of the OEC in a monomer-trimer approximation of the magnetic topology. ${ }^{62}$ Model W6-1 provides for the first time a structural realization of this mode of action. As an aside, it is worth mentioning that the above analysis can clarify an interesting technical point. Earlier theoretical work employed smaller models that did not include the crystallographic water W6 which hydrogen-bonds to $04 .{ }^{69}$ It was already noticed that the computed $\Delta E$ values for these models compared well with experimental values from methanol-treated samples, even though methanol was obviously not included in the models. If we assume that W6-1 is a correct representation of how methanol interacts with the OEC, an explanation for this curious result becomes immediately obvious: a computational model that lacks W6 mimics the effect of methanol on the hydrogen bonding environment of $\mathrm{O} 4$.

Evaluation in terms of magnetic interactions allows us to eliminate most models, including all those that involve direct
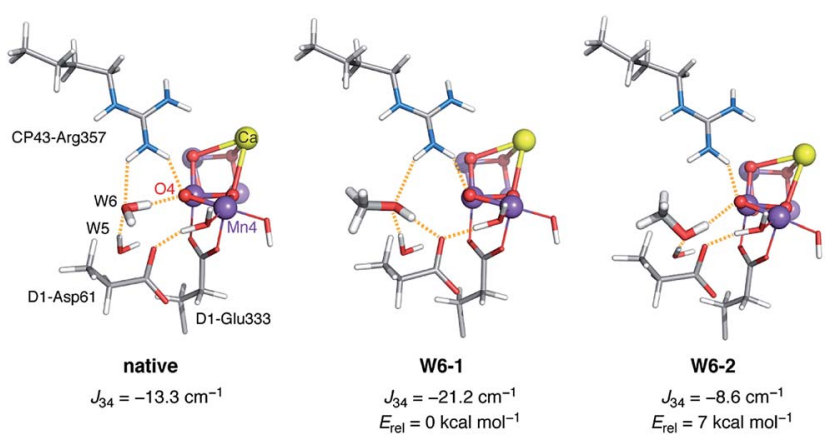

Fig. 4 Comparison of the local hydrogen-bonding environment of $\mathrm{O} 4$ in the native system (left) and in the two models W6-1 and W6-2 with methanol occupation of the W6 site.
$\mathrm{MeOH}$ ligation to $\mathrm{Mn}$. Still, this criterion is not sufficient to definitively distinguish between direct binding of methanol to $\mathrm{Ca}$ (model W4-1d) or second-sphere interaction in the vicinity of O4 (model W6-1). In the following section this distinction is achieved conclusively by comparison of the computed ${ }^{13} \mathrm{C}$ hyperfine coupling parameters with the data of Oyala et al. ${ }^{65}$

\section{${ }^{13} \mathrm{C}$ hyperfine interactions}

After confirming that our predictions for ${ }^{13} \mathrm{C}$ hyperfine interactions are reliable by comparing with the same reference mixed-valence synthetic complex ${ }^{113}$ as the one used in the experimental study ${ }^{65}$ (Fig. $\mathrm{S} 1 \dagger$ ), we have computed the relevant parameters for all models of Fig. 3 and Table 2 lists the isotropic coupling $A_{\text {iso }}$ and the dipolar coupling $T$ for the ${ }^{13} \mathrm{C}$ nucleus of methanol in all structures. Even though most models were already disfavored based on the energy level splittings in the previous section, it is important to discuss the ${ }^{13} \mathrm{C}$ hyperfine parameters of the group of models that involve methanol ligation to Mn4, because the two properties lead to identical conclusions and strongly reinforce each other. The ${ }^{13} \mathrm{C} A_{\text {iso }}$ values for $\mathrm{MeO}^{-}$binding are two orders of magnitude larger than the experimental values $(5.73 \mathrm{MHz}$ and $4.06 \mathrm{MHz}$ for W1-1d and W2-2d), and even the "best-case" scenario represented by model W1-1 is off by an order of magnitude. The deviation of computed dipolar coupling $T$ values from experiment is equally dramatic, with values ranging from $1.53 \mathrm{MHz}$ (W2-1) up to $2.96 \mathrm{MHz}$ (W1-1d). In view of the above, the conclusion that methanol does not coordinate to Mn4 is definitive.

With respect to the calcium site, models W3-1 and W4-1 agree very well with the ${ }^{13} \mathrm{C}$ hyperfine data, and this observation is in agreement with the conclusions of Oyala et al., who favored calcium as one of the likely methanol interaction sites. ${ }^{65} \mathrm{~W} 3-1$, together with W6-1 discussed below, show the best overall agreement with the experimental values. On the other hand, both W3-1 and W4-1 were excluded in the previous section because they cannot explain the increase in the energy gap $\Delta E$, thus these models cannot satisfy both constraints. The two models that were favored instead were those that involved

Table $2{ }^{13} \mathrm{C}$ methanol isotropic and dipolar hyperfine coupling parameters $(\mathrm{MHz})$ of all models, compared with experimental values for the $\mathrm{S}_{2}$ state of the OEC in spinach

\begin{tabular}{llllll}
\hline Model & $\left|A_{\text {iso }}\right|$ & $|T|$ & Model & $\left|A_{\text {iso }}\right|$ & $|T|$ \\
\hline W1-1 & 0.25 & 1.82 & W6-2 & 0.16 & 0.65 \\
W1-1d & 5.73 & 2.96 & W7-1 & 0.02 & 0.36 \\
W2-1 & 1.06 & 1.53 & W8-1 & 0.00 & 0.17 \\
W2-1d & 4.06 & 2.54 & $\mathbf{W 8 - 2}$ & 0.34 & 0.59 \\
W3-1 & 0.01 & 0.28 & W9-1 & 0.01 & 0.20 \\
W3-1d & 3.03 & 2.45 & $\mathbf{W 1 0 - 1}$ & 0.00 & 0.14 \\
W4-1 & 0.03 & 0.42 & $\mathbf{W 1 0 - 2}$ & 1.37 & 0.40 \\
W4-1d & 14.11 & 1.44 & $\mathbf{W 1 1 - 1}$ & 1.35 & 0.73 \\
W5-1 & 0.14 & 0.49 & $\mathbf{W 1 2 - 1}$ & 0.01 & 0.58 \\
W6-1 & 0.02 & 0.24 & $\mathbf{W 1 2 - 2}$ & 0.02 & 0.76 \\
Exp. & $0.05 \pm 0.02$ & $0.27 \pm 0.05$ & Exp. & $0.05 \pm 0.02$ & $0.27 \pm 0.05$
\end{tabular}


binding of deprotonated methanol, W3-1d and W4-1d, the latter being one of the two strong candidates. However, the computed values are compelling: this type of methanol binding cannot possibly reproduce the experimental ${ }^{13} \mathrm{C}$ hyperfine data, because both $A_{\text {iso }}$ and $T$ are orders of magnitude too large.

Combined with the results of the previous section this leaves only one candidate, model W6-1. In stark contrast to W4-1d, the agreement with experiment in this case is excellent, with $A_{\text {iso }}=0.02$ versus the experimental value of $0.05 \pm 0.02$ and $T=0.24$ versus the experimental value of $0.27 \pm 0.05$. Therefore, there is only one model, W6-1, requiring methanol delivery through the water channel associated with $\mathrm{O} 4$, that simultaneously accounts for the effect on the magnetic couplings of the OEC in the $S_{2}$ state and reproduces the experimental ${ }^{13} \mathrm{C}$ hyperfine data.

\section{Possibility of a secondary binding site}

Although the identification of W6 as the site occupied by methanol is definitive, one may consider whether a second molecule of methanol can be present at a secondary site, potentially depending on concentration. Based on the experimental data and our calculations, binding as a first-sphere ligand to Mn and as a methoxy to calcium are excluded, while occupation of the W6 site is required to explain simultaneously the effect on magnetism and the ${ }^{13} \mathrm{C}$ hyperfine data, so if there is only one way that methanol interacts with the OEC, this is represented by model W6-1. On the other hand, the presence of a second molecule of methanol cannot be excluded if it occupies a site that satisfies the ${ }^{13} \mathrm{C}$ hyperfine data while not adversely affecting the energy level splitting. A hypothesis regarding the presence of two methanol binding sites was previously advanced by Sjöholm et al. to account for the different methanol concentration dependence in the induction of "split" EPR signals of the OEC, that is, of states that contain a tyrosyl radical interacting with the manganese cluster. ${ }^{63}$ The postulated interaction modes (direct binding of methanol to Mn1 and/or Mn4) can no longer be considered viable, so it might be worth revisiting that analysis in light of the present results and of the required occupation of the W6 site.

\section{S-state invariance of the methanol binding pocket}

The $S_{1}$ state of the OEC is diamagnetic $(S=0)$. Parallel-mode EPR however probes the first excited state $(S=1)^{\mathbf{4 8 , 1 1 4 , 1 1 5}}$ which is very low in energy, less than $2 \mathrm{~cm}^{-1}$ higher than the ground state. ${ }^{48}$ In terms of methanol interaction, it was shown that the parallel-mode $S_{1}$ state signal at $g \approx 4.9$ is no longer observable upon addition of methanol, ${ }^{48}$ suggesting that the triplet state is depopulated, i.e. the energy splitting $\Delta E$ between the ground and first excited states is increased. If we assume that the methanol binding pocket in the $S_{1}$ state is the same as in $S_{2}$ and is represented well by the $S_{1}$ analogue of model W6-1, these observations obtain an obvious structure-based rationalization that mirrors the analysis we presented for the $S_{2}$ state. The antiferromagnetic coupling between Mn3 and Mn4 in the $\mathrm{S}_{1}$ state $^{72}$ would be enhanced by the presence of methanol via the same mechanism discussed above, stabilizing the $S=0$ ground state and resulting in disappearance of the excited-state $S=1$ signal.

Given that the oxidation states of the manganese cluster cannot influence the channel architecture, methanol should access the OEC through the same channel regardless of the catalytic state of the cluster, so we also expect the same methanol site in the $S_{0}$ and $S_{3}$ states. The phenomenology of split EPR signals of both the $S_{1} Y_{Z}$ and the $S_{0} Y_{Z} \cdot$ states has been rationalized in terms of an increase in $\Delta E$ upon addition of methanol, ${ }^{60}$ so a similar mode of interaction as for the $S_{1}$ and $S_{2}$ states (operating on the Mn3-Mn4 antiferromagnetic coupling) would be valid for the $S_{0}$ state as well. We prefer not to propose specific $\mathrm{S}_{0}$ models at this point because of the more complex EPR phenomenology of this state $^{50,77,116,117}$ and remaining uncertainties in the protonation states of terminal waterderived ligands and oxo bridges..$^{35,72,118-120}$ EPR studies of the $\mathrm{S}_{3}$ state reveal a rich and complex phenomenology. ${ }^{53,54,58,71,121-133} \mathrm{~S}_{3}$ is an integer spin state and interpretation of spectroscopic data is additionally complicated by heterogeneity, related at least in part to structural polymorphism that has only recently began to be understood..$^{73,134}$ A more complete understanding of the $\mathrm{S}_{3}$ state is necessary before one can confidently discuss methanol interaction in terms of atomic structure. Nevertheless, the present work constrains the methanol interaction site to W6 and restricts the question only to how methanol might affect the different possible $S_{3}$ forms, or stabilize one of these forms over the others, for example by inhibiting water delivery.

In conclusion, methanol likely occupies the same site in all states, adopts approximately the same orientation and, at least in the $\mathrm{S}_{0}-\mathrm{S}_{2}$ states, affects the electronic structure in the same fashion. Finally, we would like to point out that since direct ligation to a $\mathrm{Mn}$ ion is excluded, the apparent S state dependent sensitivity to methanol cannot be attributed to interaction with Mn ions in different oxidation states. An alternative and simpler interpretation of such observations emerges from the present work: if methanol can occupy the same site in all S states and have a similar effect on the electronic structure by modulating the Mn3-O4-Mn4 interaction, the apparent differences in sensitivity between catalytic states arise simply from the different weight of $J_{34}$ in determining the magnetic properties of the cluster in each $\mathrm{S}$ state.

\section{Effect on the two components of the $S_{2}$ state}

Two EPR signals are associated with the $S_{2}$ state of the OEC in plants, a multiline signal centered at $g \approx 2$ that corresponds to an $S=1 / 2$ ground state species, and a signal at $g \approx 4.1$ that arises from an $S=5 / 2$ form of the cluster. In 2012 we demonstrated that these two signals correspond to two geometric and valence isomers that are interconvertible and lie very close in energy, within $1 \mathrm{kcal} \mathrm{mol}^{-1}$ or less. ${ }^{70}$ The $S=1 / 2$ signal arises from the most stable open-cubane form of the cluster (with respect to the position of the $\mathrm{O} 5$ bridge) that has the oxidation state distribution III-IV-IV-IV, i.e. the unique Mn(III) ion is Mn1. The $S=5 / 2$ species adopts instead a closedcubane form with the Mn(III) ion located at the "dangler" Mn4 site (Scheme 1). 


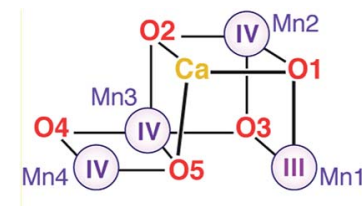

$$
S=1 / 2, g \approx 2.0
$$

Scheme 1

A crucial experimental observation is that in PS-II from plants, methanol prevents formation of the $g \approx 4.1$ signal, that is, it destabilizes the valence isomer that contains a $\mathrm{Mn}$ (III) at the Mn4 site. The reorganization of the hydrogen bonding described above for model W6-1 provides a possible structurebased explanation of the phenomenology: the energetically favorable removal of the $\mathrm{H}$-bonding interaction with $\mathrm{O} 4$ when methanol occupies the W6 site results in a more negatively charged $\mathrm{O} 4$ bridge, which in turn favors the +IV oxidation state for the directly ligated Mn4, shifting the equilibrium from the closed-cubane isomer that contains a Mn4(III) towards the opencubane, $S=1 / 2$, Mn4(Iv) isomer associated with the multiline signal.

\section{Species dependence}

A historically persistent question that relates to the methanol effect is the species-dependent response. Specifically, the influence of methanol on the EPR spectra and the magnetic coupling is reported to be more pronounced in higher plants than in cyanobacteria. ${ }^{62}$ The present work converges to an atomistic model that is consistent with observations (particularly those from spinach samples), but cannot directly explain why the methanol effect is less pronounced in cyanobacteria. No species-dependent substitutions are known for any of the amino acid residues included in our QM model and hence it is reasonable to expect that model W6-1 should apply equally well to all organisms. It is improbable that fundamentally different access modes exists between species, therefore we consider the following explanation as most likely: the model favored in the present work must be equally valid for both cyanobacteria and higher plants, if methanol can reach this site and adopt this position in all organisms. In the following we identify the structural origin of the species-dependent response and we demonstrate that it strongly supports the proposed delivery channel.

Sequence alignment for the four core proteins of T. vulcanus and $S$. oleracea (spinach) PS-II reveals in total 41 differences in D1 (PsbA), 34 in D2 (PsbD), 73 in CP43 (PsbC), and 114 in CP47 (PsbB). The vast majority are conservative substitutions; nonconservative substitutions are found mostly in the CP proteins (detailed sequence alignments are provided in the ESI $\dagger$ ). Overall homology is approximately $88 \%$ and $91 \%$ for the D1 and D2 chains, while if conservative substitutions are not taken into account, the sequence similarity rises to $96 \%$. Inspection of the sites with respect to the crystallographic model of PS-II from $T$. vulcanus $^{7,8}$ shows that most differences occur in the flexible outer loops of the D1/D2 proteins, or in the periphery of transmembrane helices of the CP43/CP47 proteins and the lumenal side of CP47 (Fig. S2 $\dagger$ ). Consistent with the strictly conserved nature of the OEC and the identical chemistry across all oxygenic photosynthetic organisms, no variants exist in the first coordination sphere (associated with the D1 and CP43 proteins), in the environment of the redox active tyrosine $\mathrm{Y}_{\mathrm{Z}}$ (D1-Y161), or in any parts of the protein that either bond covalently or interact via direct or water-mediated hydrogen bonding with the immediate environment of the OEC.

Within a sphere of $10 \AA$ radius around any atom of the $\mathrm{Mn}_{4} \mathrm{CaO}_{5}$ cluster, we find two differences, both in the D1 protein: cyanobacterial Ser85 is threonine in spinach PS-II (a conservative substitution that has no relevance to any of the methanol interaction models), and cyanobacterial Asn87 is alanine in spinach (note that T. vulcanus and T. elongatus are identical in this respect). The latter substitution is distinctly non-conservative: it involves side chains of markedly different size and properties. No other such difference between the two organisms could be found along any of the channels in a radius of $c a .20 \AA$ around the OEC for the core proteins. The unique importance of the N87A substitution is that it occurs right at the final turn of the O4-related channel we identified as the delivery channel for methanol.

Focusing on the local structure of this site (Fig. 5), it is apparent that cyanobacterial D1-Asn87 forms a hydrogen bond with the backbone of CP43-Glu354 (the residue that bridges Mn2 and Mn3), whereas in spinach D1-Ala87 cannot form such a bond. The difference in the formation of this hydrogen bond and the difference in the size of the side chains means that the diameter and local architecture of the channel at this point are also different: in the D1-Ala87 analogue methanol can access the interaction site unimpeded and adopt the energetically favorable conformation that corresponds to model W6-1. In this sense, W6-1 most probably reflects better how methanol interacts in spinach, something that explains why our QM models for methanol interaction agree better with experimental data from spinach than from cyanobacteria. By contrast, in the D1-Asn87 analogue the larger side chain of asparagine may sterically interact with the W6 pocket, while the formation of
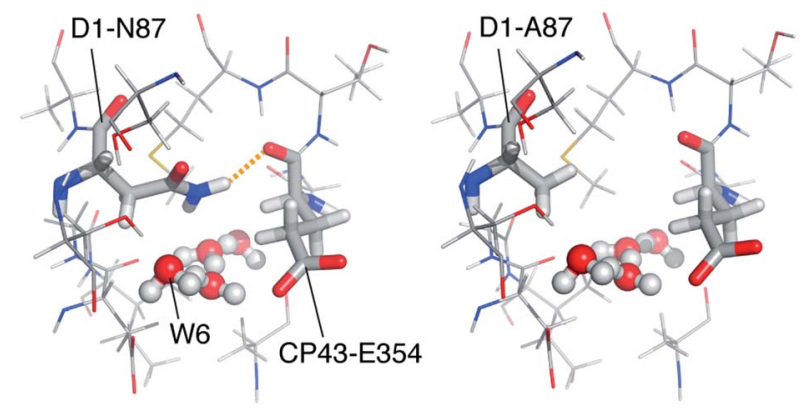

Fig. 5 The water channel with the critical N87A substitution, viewed from the OEC. Coordinates for the cyanobacterial model (left) are taken from a QM/MM mode ${ }^{17}$ that was based on the crystallographic model of PS-II from T. vulcanus. ${ }^{8}$ The model for spinach (right) was constructed by local side chain replacement to demonstrate the expected structural effect. 
a hydrogen bond with CP43-Glu354 results in a reduced channel diameter and restricted accessibility. This might impose a different orientation of methanol than in model W6-1, or most probably prohibit access of methanol to the W6 site altogether. Therefore, W6-1 is a good model for methanol interaction in plants, while a model with a more remote placement of methanol along the same water channel (e.g. a variant of W5-1 or a site even more remote than W5) would better represent methanol interaction in cyanobacteria.

Computational studies using multiscale approaches that involve dynamics simulations with extended models of PS-II would be necessary to determine the intrinsic differences between the two species with atomic detail; such models are currently under development in our group. Within the context of the present work, the above analysis is sufficient to establish that: (a) the only relevant difference in protein sequences between cyanobacteria and spinach is the D1-N87A substitution at the channel identified as responsible for delivery of methanol and at a position close to the W6 site, and (b) the presence of D1-Ala87 in spinach should result in increased accessibility of the W6 site to methanol compared to cyanobacterial PS-II, where access to the W6 site may be severely restricted or impossible.

\section{Implications for regulation and catalysis}

The model for methanol interaction proposed here requires access from the water channel associated with the $\mathrm{O} 4$ bridge of the cluster. In the literature this channel is referred to as the "narrow" channel by Ho and Styring, ${ }^{20}$ "E, F" in the work of Gabdulkhakov et al., "channel 2" by Vassiliev et al. ${ }^{25}$ and "Path 3 " by Ogata et al. ${ }^{30}$ Consistent with the present findings, Ho and Styring had already suggested by analysis of a low-resolution static crystallographic model of PS-II that this channel would be the most permeable to methanol. ${ }^{20}$ Molecular dynamics simulations by Vassiliev et al. subsequently demonstrated that: (a) this channel connects the OEC directly with the lumenal surface of PS-II at the cavity formed by the extrinsic PsbO and PsbU proteins, and (b) this channel has the lowest free energy barrier for water permeation (peak activation energy ca. $9 \mathrm{kcal} \mathrm{mol}^{-1}$ ) than all other channels identified. ${ }^{25}$ Moreover, recent experimental and theoretical work has demonstrated that $\mathrm{NH}_{3}$ binds to Mn4 in the place of $\mathrm{W} 1$ in the $\mathrm{S}_{2}$ state of the OEC. ${ }^{\mathbf{4 4 , 6 4 , 6 6 , 8 3}}$ Although the interaction modes of $\mathrm{NH}_{3}$ and $\mathrm{MeOH}$ are fundamentally different - the first one acting as a direct ligand to Mn4, the latter interacting from the second coordination sphere-access of methanol coincides with access of ammonia. This is consistent with competition studies that showed interrelations between the binding of these substrate analogues ${ }^{56}$ and reinforces the assignment of the $\mathrm{O} 4$ channel as the one involved in delivery of substrate analogues, and, by extension, of substrate water.

The $\mathrm{O} 4$ water channel was recently proposed instead to be involved in proton transfer, at least in the $\mathrm{S}_{0}-\mathrm{S}_{1}$ transition, if the critical assumption is made that $\mathrm{O} 4$ is protonated in the $\mathrm{S}_{0}$ state. ${ }^{35,135}$ Although this idea is not implausible, ${ }^{\mathbf{1 3 5}}$ it seems to us improbable for three reasons: (a) explicit calculation of protonation patterns by a wide variety of methods $s^{72,118,119}$ suggests that $\mathrm{O} 4$ protonation is unfavorable in $\mathrm{S}_{0}$, therefore the basis for these computational constructs is questionable; (b) the involvement of this channel in the delivery of methanol (identified here), of ammonia (as a ligand to Mn4), ${ }^{\mathbf{4 4 , 6 4 , 6 6 , 8 3}}$ as well as of water in the $S_{3}$ state $^{73}$ would necessitate an unlikely dual role for this channel; and (c) residue D1-Asn87 would have to be involved in the hypothetical proton transfer, so models that might be valid for T. vulcanus would not be transferable to D1-Ala87 organisms, which is implausible for a function as critical as proton removal from the water oxidation site. Proton egress instead is most likely to occur via the other channel associated with D1-Asp61, as previously assigned by Knapp and co-workers based on the monotonic increase of calculated $\mathrm{p} K_{\mathrm{a}}$ values of titratable residues, ${ }^{34}$ and by FTIR and mutation studies. ${ }^{\mathbf{1 3 6 , 1 3 7}}$ The $\mathrm{H}_{2} \mathrm{O}$ molecule occupying the W1 site in the $\mathrm{S}_{2}$ state has been identified as the Mn-bound ligand that is

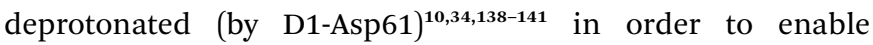
oxidation of Mn4 and allow transition to the $S_{3}$ state. ${ }^{73,132,142-144}$ In this case, the influence of $\mathrm{MeOH}$ on proton release from $\mathrm{W} 1$ would be a second-order effect, acting through perturbation of the proton acceptor. This may result in modulation of the relative stabilities of intermediates such as tyrosyl radical states $^{53,57,58,63,122,145,146}$ in a species-dependent manner and is a subject that deserves further study.

The lack of experimental or theoretical support for either $\mathrm{NH}_{3}$ or $\mathrm{MeOH}$ interacting with $\mathrm{Ca}^{2+}$ suggests that the calcium channel system may not be involved in small molecule access to the OEC. It has been proposed instead that the channels at this side of the cluster are more hydrophobic and hence better optimized for removal of dioxygen, ${ }^{\mathbf{2 0 , 2 6}}$ and that calcium itself may play a role in facilitating $\mathrm{O}_{2}$ release from the cluster. ${ }^{147}$ In this case, $\mathrm{Ca}^{2+}$ is not a likely site of substrate binding and by extension the water ligands of $\mathrm{Ca}^{2+}$ (W3, W4) are not likely to be substrates in $\mathrm{O}-\mathrm{O}$ bond formation. Substrate analogues are instead found either on Mn4 or at the endpoint of the $\mathrm{O} 4$ channel, implying that this channel may be the only one involved in substrate delivery and that Mn4 may be the only site involved in substrate binding. This is consistent with recent studies of the $\mathrm{S}_{2}-\mathrm{S}_{3}$ progression, ${ }^{73,134,142,148}$ which showed that the first component of the $\mathrm{S}_{3}$ state is a "closed-cubane" structure with a five-coordinate Mn4(Iv) center. ${ }^{73}$ The approximately trigonal bipyramidal geometry of the Mn4(Iv) site of this species results in properties such as absorption in the near-IR and high zero-field splitting, ${ }^{73}$ similarly to analogous synthetic $\mathrm{Mn}$ (Iv) complexes. ${ }^{149-151}$ These studies established that water binding can only occur at this $\mathrm{S}_{3}$-state species: water is delivered via the $\mathrm{O} 4$ channel to the Mn4 center and binds via a low-barrier transition state, ${ }^{73,148}$ allowing access to the other components of the $\mathrm{S}_{3}$ state. ${ }^{71}$ Based on the above, we propose that occupation of the W6 site by methanol may enhance the $S_{3}$ population with the five-coordinate $\mathrm{Mn}$ (Iv) ion by inhibiting water delivery to the Mn4 site. ${ }^{73,148}$

Water binding to the Mn4 ion $^{73,148}$ and internal structural rearrangement ${ }^{134,152}$ would be necessary to reach the structural form of the $\mathrm{S}_{3}$ state attributed to an all-octahedral Mn(Iv) species ${ }^{71}$ that has been proposed by Siegbahn to be the active form of the OEC in $\mathrm{O}-\mathrm{O}$ bond formation, ${ }^{\mathbf{1 5}, \mathbf{1 4 3}, \mathbf{1 5 3}}$ in an oxo-oxyl coupling 
scenario. ${ }^{\mathbf{1 4 , 1 0 4 , 1 5 4 , 1 5 5}}$ Given that neither methanol nor ammonia are inhibitors and that the kinetics of substrate exchange show both substrates to be already bound in the $\mathrm{S}_{2}$ state, ${ }^{156-159}$ we believe that further evidence is required to conclusively establish whether water binding in the $S_{3} s_{a t e^{71,160,161}}$ is catalytically required for progression to the $\mathrm{S}_{3} \mathrm{Y}_{\mathrm{Z}}{ }^{\cdot}$ state and its subsequent deprotonation and transition to $S_{4}$. A better characterization of the $S_{3}$ and $S_{3} Y_{Z}{ }^{\cdot}$ states is needed to clarify this point. ${ }^{133,145,146,162-167}$

\section{Conclusions}

Precise structural characterization of how substrate analogues interact with the oxygen-evolving complex of photosystem-II is important for understanding regulatory and mechanistic aspects of water oxidation. We studied the interaction of methanol with the $\mathrm{S}_{2}$ state of the OEC using extensive quantum mechanical models evaluated against experimental data. The two main criteria used were: (a) the change in the energy splitting between the $S=1 / 2$ ground-state and the $S=3 / 2$ first excited state of the complex, ${ }^{62}$ and (b) the recently reported ${ }^{13} \mathrm{C}$ hyperfine coupling parameters of isotopically labeled methanol. ${ }^{65}$ Our results definitively exclude direct binding of methanol to manganese and strongly disfavor direct interaction with calcium. The only interaction mode that is consistent with observations involves displacement of W6, the final water molecule of the water channel associated with the $\mathrm{O} 4$ bridge of the cluster, proximal to the "dangler" Mn4 ion. This site reproduces the isotropic and dipolar ${ }^{13} \mathrm{C}$ hyperfine couplings for methanol and provides a physically transparent explanation for the observed ground-state stabilization: the preferred methanol orientation removes the hydrogen bond between $\mathrm{O} 4$ and W6, amplifying the antiferromagnetic coupling between Mn3 and Mn4. The same interaction mode can be expected in other states. The effect of removing the hydrogen bond to $\mathrm{O} 4$ can also explain the stabilization of the $S=1 / 2$ form of the $\mathrm{S}_{2}$ state in plant PS-II. Sequence alignments of PS-II core proteins from cyanobacteria and spinach reveal an important difference at the suggested delivery channel close to the methanol binding pocket, where D1-Asn87 of cyanobacteria is D1-Ala87 in spinach, a substitution that affects the accessibility of the site to methanol and can account for the species-dependence of the methanol effect. This result presents an obvious target for future research, the introduction of the D1-N87A mutation in cyanobacterial PS-II. The structural characterization of the methanol interaction site, the identification of the delivery channel and the correlation with other experimental and computational results are in line with the pivot mechanism for delivery and binding of water to the five-coordinate Mn4(Iv) ion of an $\mathrm{S}_{3}$-state component, ${ }^{73}$ and support the idea that the $\mathrm{O} 4$ channel is uniquely responsible for delivery of water and substrate analogues to the OEC, with Mn4 being the only directly solvent-accessible site of the cluster.

\section{Acknowledgements}

The authors gratefully acknowledge computing time granted by the John von Neumann Institute for Computing (NIC) and provided on the supercomputer JUROPA at Jülich Supercomputing Centre (JSC) (NIC project No. 7056). This work is supported by the Cluster of Excellence RESOLV (EXC 1069) funded by the Deutsche Forschungsgemeinschaft. Network support by the COST action CM1305 "Explicit Control Over Spin-states in Technology and Biochemistry (ECOSTBio)" is gratefully acknowledged.

\section{Notes and references}

1 V. Krewald, M. Retegan and D. A. Pantazis, Top. Curr. Chem., 2016, 371, 23-48.

2 J.-R. Shen, Annu. Rev. Plant Biol., 2015, 66, 23-48.

3 N. Cox, D. A. Pantazis, F. Neese and W. Lubitz, Acc. Chem. Res., 2013, 46, 1588-1596.

4 D. J. Vinyard, G. M. Ananyev and G. C. Dismukes, Annu. Rev. Biochem., 2013, 82, 577-606.

5 J. Messinger, T. Noguchi and J. Yano, in Molecular Solar Fuels, ed. T. J. Wydrzynski and W. Hillier, The Royal Society of Chemistry, Cambridge, 2012, pp. 163-207.

6 J. P. McEvoy and G. W. Brudvig, Chem. Rev., 2006, 106, 4455-4483.

7 M. Suga, F. Akita, K. Hirata, G. Ueno, H. Murakami, Y. Nakajima, T. Shimizu, K. Yamashita, M. Yamamoto, H. Ago and J.-R. Shen, Nature, 2014, 517, 99-103.

8 Y. Umena, K. Kawakami, J.-R. Shen and N. Kamiya, Nature, 2011, 473, 55-60.

9 B. Loll, J. Kern, W. Saenger, A. Zouni and J. Biesiadka, Nature, 2005, 438, 1040-1044.

10 K. N. Ferreira, T. M. Iverson, K. Maghlaoui, J. Barber and S. Iwata, Science, 2004, 303, 1831-1838.

11 B. Kok, B. Forbush and M. McGloin, Photochem. Photobiol., 1970, 11, 457-475.

12 P. Joliot, G. Barbieri and R. Chabaud, Photochem. Photobiol., 1969, 10, 309-329.

13 V. Krewald, F. Neese and D. A. Pantazis, Isr. J. Chem., 2015, 55, 1219-1232.

14 P. E. M. Siegbahn, Acc. Chem. Res., 2009, 42, 1871-1880.

15 P. E. M. Siegbahn, Biochim. Biophys. Acta, Bioenerg., 2013, 1827, 1003-1019.

16 T. Wydrzynski, W. Hillier and J. Messinger, Physiol. Plant., 1996, 96, 342-350.

17 M. Retegan, F. Neese and D. A. Pantazis, J. Chem. Theory Comput., 2013, 9, 3832-3842.

18 J. W. Murray and J. Barber, J. Struct. Biol., 2007, 159, 228237.

19 F. M. Ho, Photosynth. Res., 2008, 98, 503-522.

20 F. M. Ho and S. Styring, Biochim. Biophys. Acta, Bioenerg., 2008, 1777, 140-153.

$21 \mathrm{~J}$. Murray and J. Barber, in Photosynthesis. Energy from the Sun, ed. J. Allen, E. Gantt, J. Golbeck and B. Osmond, Springer Netherlands, 2008, pp. 467-470.

22 A. Gabdulkhakov, A. Guskov, M. Broser, J. Kern, F. Muh, W. Saenger and A. Zouni, Structure, 2009, 17, 1223-1234.

23 S. Vassiliev, P. Comte, A. Mahboob and D. Bruce, Biochemistry, 2010, 49, 1873-1881. 
24 F. M. Ho, in Molecular Solar Fuels, ed. T. J. Wydrzynski and W. Hillier, The Royal Society of Chemistry, Cambridge, 2012, pp. 208-248.

25 S. Vassiliev, T. Zaraiskaya and D. Bruce, Biochim. Biophys. Acta, Bioenerg., 2012, 1817, 1671-1678.

26 S. Vassiliev, T. Zaraiskaya and D. Bruce, Biochim. Biophys. Acta, Bioenerg., 2013, 1827, 1148-1155.

27 L. Vogt, D. J. Vinyard, S. Khan and G. W. Brudvig, Curr. Opin. Chem. Biol., 2015, 25, 152-158.

28 A.-N. Bondar and H. Dau, Biochim. Biophys. Acta, Bioenerg., 2012, 1817, 1177-1190.

29 K. Linke and F. M. Ho, Biochim. Biophys. Acta, Bioenerg., 2014, 1837, 14-32.

30 K. Ogata, T. Yuki, M. Hatakeyama, W. Uchida and S. Nakamura, J. Am. Chem. Soc., 2013, 135, 15670-15673.

31 These three channels may split into more branches or intersect with branches of other channels at other regions of the enzyme, having different exit/entry points. The channel architecture of PS-II as a whole is still an open field of study; in the present work we are only concerned with the well-defined immediate vicinity of the active site.

32 H. Bao, P. Dilbeck and R. Burnap, Photosynth. Res., 2013, 116, 215-229.

33 I. L. McConnell, Photosynth. Res., 2008, 98, 261-276.

34 H. Ishikita, W. Saenger, B. Loll, J. Biesiadka and E.-W. Knapp, Biochemistry, 2006, 45, 2063-2071.

35 K. Saito, A. W. Rutherford and H. Ishikita, Nat. Commun., 2015, 6, 8488.

36 W. F. Beck, J. C. De Paula and G. W. Brudvig, J. Am. Chem. Soc., 1986, 108, 4018-4022.

37 L.-E. Andréasson, Ö. Hansson and K. von Schenck, Biochim. Biophys. Acta, Bioenerg., 1988, 936, 351-360.

38 R. D. Britt, J. L. Zimmermann, K. Sauer and M. P. Klein, J. Am. Chem. Soc., 1989, 111, 3522-3532.

39 A. Boussac, A. W. Rutherford and S. Styring, Biochemistry, 1990, 29, 24-32.

40 H. Dau, J. C. Andrews, T. A. Roelofs, M. J. Latimer, W. Liang, V. K. Yachandra, K. Sauer and M. P. Klein, Biochemistry, 1995, 34, 5274-5287.

41 H.-A. Chu, Y.-W. Feng, C.-M. Wang, K.-A. Chiang and S.-C. Ke, Biochemistry, 2004, 43, 10877-10885.

42 C.-H. Fang, K.-A. Chiang, C.-H. Hung, K. Chang, S.-C. Ke and H.-A. Chu, Biochemistry, 2005, 44, 9758-9765.

43 L.-H. Hou, C.-M. Wu, H.-H. Huang and H.-A. Chu, Biochemistry, 2011, 50, 9248-9254.

44 M. Pérez Navarro, W. M. Ames, H. Nilsson, T. Lohmiller, D. A. Pantazis, L. Rapatskiy, M. M. Nowaczyk, F. Neese, A. Boussac, J. Messinger, W. Lubitz and N. Cox, Proc. Natl. Acad. Sci. U. S. A., 2013, 110, 15561-15566.

45 J. L. Zimmermann and A. W. Rutherford, Biochemistry, 1986, 25, 4609-4615.

46 G. A. Lorigan and R. D. Britt, Biochemistry, 1994, 33, 1207212076.

47 R. J. Pace, P. Smith, R. Bramley and D. Stehlik, Biochim. Biophys. Acta, Bioenerg., 1991, 1058, 161-170.

48 T. Yamauchi, H. Mino, T. Matsukawa, A. Kawamori and T.-a. Ono, Biochemistry, 1997, 36, 7520-7526.
49 D. A. Force, D. W. Randall, G. A. Lorigan, K. L. Clemens and R. D. Britt, J. Am. Chem. Soc., 1998, 120, 13321-13333.

50 K. A. Åhrling, S. Peterson and S. Styring, Biochemistry, 1998, 37, 8115-8120.

51 Z. Deák, S. Peterson, P. Geijer, K. A. Åhrling and S. Styring, Biochim. Biophys. Acta, Bioenerg., 1999, 1412, 240-249.

52 G. A. Lorigan and R. David Britt, Photosynth. Res., 2000, 66, 189-198.

53 N. Ioannidis and V. Petrouleas, Biochemistry, 2000, 39, 5246-5254.

54 N. Ioannidis and V. Petrouleas, Biochemistry, 2002, 41, 9580-9588.

55 K. A. Åhrling, M. C. W. Evans, J. H. A. Nugent and R. J. Pace, Biochim. Biophys. Acta, Bioenerg., 2004, 1656, 66-77.

56 M. C. W. Evans, R. J. Ball and J. H. A. Nugent, FEBS Lett., 2005, 579, 3081-3084.

57 V. Petrouleas, D. Koulougliotis and N. Ioannidis, Biochemistry, 2005, 44, 6723-6728.

58 N. Ioannidis, G. Zahariou and V. Petrouleas, Biochemistry, 2006, 45, 6252-6259.

59 K. A. Åhrling, M. C. W. Evans, J. H. A. Nugent, R. J. Ball and R. J. Pace, Biochemistry, 2006, 45, 7069-7082.

60 J.-H. Su, K. G. V. Havelius, F. Mamedov, F. M. Ho and S. Styring, Biochemistry, 2006, 45, 7617-7627.

61 B. Nöring, D. Shevela, G. Renger and J. Messinger, Photosynth. Res., 2008, 98, 251-260.

62 J.-H. Su, N. Cox, W. Ames, D. A. Pantazis, L. Rapatskiy, T. Lohmiller, L. V. Kulik, P. Dorlet, A. W. Rutherford, F. Neese, A. Boussac, W. Lubitz and J. Messinger, Biochim. Biophys. Acta, Bioenerg., 2011, 1807, 829-840.

63 J. Sjöholm, G. Chen, F. Ho, F. Mamedov and S. Styring, Biochemistry, 2013, 52, 3669-3677.

64 T. Lohmiller, V. Krewald, M. Pérez Navarro, M. Retegan, L. Rapatskiy, M. M. Nowaczyk, A. Boussac, F. Neese, W. Lubitz, D. A. Pantazis and N. Cox, Phys. Chem. Chem. Phys., 2014, 16, 11877-11892.

65 P. H. Oyala, T. A. Stich, J. A. Stull, F. Yu, V. L. Pecoraro and R. D. Britt, Biochemistry, 2014, 53, 7914-7928.

66 P. H. Oyala, T. A. Stich, R. J. Debus and R. D. Britt, J. Am. Chem. Soc., 2015, 137, 8829-8837.

67 D. A. Pantazis, M. Orio, T. Petrenko, S. Zein, E. Bill, W. Lubitz, J. Messinger and F. Neese, Chem.-Eur. J., 2009, 15, 5108-5123.

68 D. A. Pantazis, M. Orio, T. Petrenko, S. Zein, W. Lubitz, J. Messinger and F. Neese, Phys. Chem. Chem. Phys., 2009, 11, 6788-6798.

69 W. Ames, D. A. Pantazis, V. Krewald, N. Cox, J. Messinger, W. Lubitz and F. Neese, J. Am. Chem. Soc., 2011, 133, 19743-19757.

70 D. A. Pantazis, W. Ames, N. Cox, W. Lubitz and F. Neese, Angew. Chem., Int. Ed., 2012, 51, 9935-9940.

71 N. Cox, M. Retegan, F. Neese, D. A. Pantazis, A. Boussac and W. Lubitz, Science, 2014, 345, 804-808.

72 V. Krewald, M. Retegan, N. Cox, J. Messinger, W. Lubitz, S. DeBeer, F. Neese and D. A. Pantazis, Chem. Sci., 2015, 6, 1676-1695. 
73 M. Retegan, V. Krewald, F. Mamedov, F. Neese, W. Lubitz, N. Cox and D. A. Pantazis, Chem. Sci., 2016, 7, 72-84.

74 V. Krewald, M. Retegan, F. Neese, W. Lubitz, D. A. Pantazis and N. Cox, Inorg. Chem., 2016, 55, 488-501.

75 A. Haddy, Photosynth. Res., 2007, 92, 357-368.

76 A. Boussac, H. Kuhl, S. Un, M. Rögner and A. W. Rutherford, Biochemistry, 1998, 37, 8995-9000.

77 A. Boussac, H. Kuhl, E. Ghibaudi, M. Rögner and A. W. Rutherford, Biochemistry, 1999, 38, 11942-11948.

78 A. Boussac, Y. Deligiannakis and A. W. Rutherford, in Photosynthesis: Mechanisms and Effects, ed. G. Garab, Kluwer Academic Publishers, Dordrecht, 1998, vol. II, pp. 1233-1240.

79 V. L. Pecoraro, M. J. Baldwin, M. T. Caudle, W.-Y. Hsieh and N. A. Law, Pure Appl. Chem., 1998, 70, 925-929.

$80 \mathrm{~J}$. S. Vrettos, J. Limburg and G. W. Brudvig, Biochim. Biophys. Acta, Bioenerg., 2001, 1503, 229-245.

81 J. P. McEvoy and G. W. Brudvig, Phys. Chem. Chem. Phys., 2004, 6, 4754-4763.

82 E. M. Sproviero, J. A. Gascon, J. P. McEvoy, G. W. Brudvig and V. S. Batista, J. Am. Chem. Soc., 2008, 130, 3428-3442.

83 J. Schraut and M. Kaupp, Chem.-Eur. J., 2014, 20, 73007308.

84 F. Neese, Wiley Interdiscip. Rev.: Comput. Mol. Sci., 2012, 2, 73-78.

85 J. P. Perdew, Phys. Rev. B, 1986, 33, 8822-8824.

86 A. D. Becke, Phys. Rev. A: At., Mol., Opt. Phys., 1988, 38, 3098-3100.

87 S. Grimme, J. Antony, S. Ehrlich and H. Krieg, J. Chem. Phys., 2010, 132, 154104.

88 A. Klamt and D. Schüürman, J. Chem. Soc., Perkin Trans. 2, 1993, 799-805.

89 E. van Lenthe, E. J. Baerends and J. G. Snijders, J. Chem. Phys., 1993, 99, 4597-4610.

90 E. van Lenthe, E. J. Baerends and J. G. Snijders, J. Chem. Phys., 1994, 101, 9783-9792.

91 C. van Wüllen, J. Chem. Phys., 1998, 109, 392-399.

92 D. A. Pantazis, X. Y. Chen, C. R. Landis and F. Neese, J. Chem. Theory Comput., 2008, 4, 908-919.

93 F. Weigend and R. Ahlrichs, Phys. Chem. Chem. Phys., 2005, 7, 3297-3305.

94 A. Schäfer, C. Huber and R. Ahlrichs, J. Chem. Phys., 1994, 100, 5829-5835.

95 F. Weigend, Phys. Chem. Chem. Phys., 2006, 8, 1057-1065.

96 V. N. Staroverov, G. E. Scuseria, J. Tao and J. P. Perdew, J. Chem. Phys., 2003, 119, 12129-12137.

97 M. Orio, D. A. Pantazis and F. Neese, Photosynth. Res., 2009, 102, 443-453.

98 M. Orio, D. A. Pantazis, T. Petrenko and F. Neese, Inorg. Chem., 2009, 48, 7251-7260.

99 V. Krewald, F. Neese and D. A. Pantazis, J. Am. Chem. Soc., 2013, 135, 5726-5739.

100 F. Neese, J. Chem. Phys., 2003, 118, 3939-3948.

101 M. A. Larkin, G. Blackshields, N. P. Brown, R. Chenna, P. A. McGettigan, H. McWilliam, F. Valentin, I. M. Wallace, A. Wilm, R. Lopez, J. D. Thompson,
T. J. Gibson and D. G. Higgins, Bioinformatics, 2007, 23, 2947-2948.

102 M. Goujon, H. McWilliam, W. Li, F. Valentin, S. Squizzato, J. Paern and R. Lopez, Nucleic Acids Res., 2010, 38, W695W699.

103 P. E. M. Siegbahn and T. Borowski, Acc. Chem. Res., 2006, 39, 729-738.

104 P. E. M. Siegbahn, Chem.-Eur. J., 2008, 14, 8290-8302.

105 P. E. M. Siegbahn and F. Himo, Wiley Interdiscip. Rev.: Comput. Mol. Sci., 2011, 1, 323-336.

106 P. E. M. Siegbahn and M. R. A. Blomberg, J. Chem. Theory Comput., 2014, 10, 268-272.

107 P. E. M. Siegbahn, Phys. Chem. Chem. Phys., 2014, 16, 11893-11900.

108 X. Wei, X. Su, P. Cao, X. Liu, W. Chang, M. Li, X. Zhang and Z. Liu, Nature, 2016, 534, 69-74.

109 M. J. Baldwin, T. L. Stemmler, P. J. Riggs-Gelasco, M. L. Kirk, J. E. Penner-Hahn and V. L. Pecoraro, J. Am. Chem. Soc., 1994, 116, 11349-11356.

110 M. J. Baldwin, N. A. Law, T. L. Stemmler, J. W. Kampf, J. E. Penner-Hahn and V. L. Pecoraro, Inorg. Chem., 1999, 38, 4801-4809.

111 D. A. Pantazis, V. Krewald, M. Orio and F. Neese, Dalton Trans., 2010, 39, 4959-4967.

112 V. Krewald, B. Lassalle-Kaiser, T. T. Boron, C. J. Pollock, J. Kern, M. A. Beckwith, V. K. Yachandra, V. L. Pecoraro, J. Yano, F. Neese and S. DeBeer, Inorg. Chem., 2013, 52, 12904-12914.

113 E. Larson, A. Haddy, M. L. Kirk, R. H. Sands, W. E. Hatfield and V. L. Pecoraro, J. Am. Chem. Soc., 1992, 114, 6263-6265.

114 S. L. Dexheimer and M. P. Klein, J. Am. Chem. Soc., 1992, 114, 2821-2826.

115 D. Koulougliotis, C. Teutloff, Y. Sanakis, W. Lubitz and V. Petrouleas, Phys. Chem. Chem. Phys., 2004, 6, 4859-4863.

116 J. Messinger, J. H. A. Nugent and M. C. W. Evans, Biochemistry, 1997, 36, 11055-11060.

117 J. Messinger, J. H. Robblee, W. O. Yu, K. Sauer, V. K. Yachandra and M. P. Klein, J. Am. Chem. Soc., 1997, 119, 11349-11350.

118 R. Pal, C. F. A. Negre, L. Vogt, R. Pokhrel, M. Z. Ertem, G. W. Brudvig and V. S. Batista, Biochemistry, 2013, 52, 7703-7706.

119 M. Amin, L. Vogt, W. Szejgis, S. Vassiliev, G. W. Brudvig, D. Bruce and M. R. Gunner, J. Phys. Chem. B, 2015, 119, 7366-7377.

120 A. Galstyan, A. Robertazzi and E. W. Knapp, J. Am. Chem. Soc., 2012, 134, 7442-7449.

121 T. Matsukawa, H. Mino, D. Yoneda and A. Kawamori, Biochemistry, 1999, 38, 4072-4077.

122 N. Ioannidis, J. H. A. Nugent and V. Petrouleas, Biochemistry, 2002, 41, 9589-9600.

123 A. Boussac, M. Sugiura, Y. Inoue and A. W. Rutherford, Biochemistry, 2000, 39, 13788-13799.

124 Y. Sanakis, J. Sarrou, G. Zahariou and V. Petrouleas, in Photosynthesis: Energy from the Sun, ed. J. F. Allen, E. Gantt, J. H. Golbeck and B. Osmond, Springer, Dordrecht, 2008, pp. 479-482. 
125 J.-H. Su, K. G. V. Havelius, F. M. Ho, G. Han, F. Mamedov and S. Styring, Biochemistry, 2007, 46, 10703-10712.

126 G. Han, F. M. Ho, K. G. V. Havelius, S. F. Morvaridi, F. Mamedov and S. Styring, Biochim. Biophys. Acta, Bioenerg., 2008, 1777, 496-503.

127 A. Boussac, M. Sugiura, A. W. Rutherford and P. Dorlet, J. Am. Chem. Soc., 2009, 131, 5050-5051.

128 M. Chrysina, G. Zahariou, N. Ioannidis and V. Petrouleas, Biochim. Biophys. Acta, Bioenerg., 2010, 1797, 487-493.

129 M. Chrysina, G. Zahariou, Y. Sanakis, N. Ioannidis and V. Petrouleas, J. Photochem. Photobiol., B, 2011, 104, 72-79.

130 K. G. V. Havelius, J.-H. Su, G. Han, F. Mamedov, F. M. Ho and S. Styring, Biochim. Biophys. Acta, Bioenerg., 2011, 1807, 11-21.

131 G. Chen, G. Han, E. Göransson, F. Mamedov and S. Styring, Biochemistry, 2012, 51, 138-148.

132 M. Retegan, N. Cox, W. Lubitz, F. Neese and D. A. Pantazis, Phys. Chem. Chem. Phys., 2014, 16, 11901-11910.

133 A. Boussac, A. W. Rutherford and M. Sugiura, Biochim. Biophys. Acta, Bioenerg., 2015, 1847, 576-586.

134 M. Capone, D. Bovi, D. Narzi and L. Guidoni, Biochemistry, 2015, 54, 6439-6442.

135 T. Takaoka, N. Sakashita, K. Saito and H. Ishikita, J. Phys. Chem. Lett., 2016, 7, 1925-1932.

136 R. J. Service, W. Hillier and R. J. Debus, Biochemistry, 2014, 53, 1001-1017.

137 R. J. Debus, Biochim. Biophys. Acta, Bioenerg., 2015, 1847, 19-34.

138 H. Dau and M. Haumann, Coord. Chem. Rev., 2008, 252, 273-295.

139 I. Rivalta, M. Amin, S. Luber, S. Vassiliev, R. Pokhrel, Y. Umena, K. Kawakami, J. R. Shen, N. Kamiya, D. Bruce, G. W. Brudvig, M. R. Gunner and V. S. Batista, Biochemistry, 2011, 50, 6312-6315.

140 R. J. Debus, Biochemistry, 2014, 53, 2941-2955.

141 P. L. Dilbeck, H. J. Hwang, I. Zaharieva, L. Gerencser, H. Dau and R. L. Burnap, Biochemistry, 2012, 51, 1079-1091.

142 D. Narzi, D. Bovi and L. Guidoni, Proc. Natl. Acad. Sci. U. S. A., 2014, 111, 8723-8728.

143 P. E. M. Siegbahn, Phys. Chem. Chem. Phys., 2012, 14, 48494856.

144 A. Klauss, M. Haumann and H. Dau, Proc. Natl. Acad. Sci. U. S. A., 2012, 109, 16035-16040.

145 G. Zahariou, M. Chrysina, V. Petrouleas and N. Ioannidis, FEBS Lett., 2014, 588, 1827-1831.
146 G. Zahariou and N. Ioannidis, Photosynth. Res., 2016, DOI: 10.1007/s11120-11016-10274-11126.

147 S. Bang, Y.-M. Lee, S. Hong, K.-B. Cho, Y. Nishida, M. S. Seo, R. Sarangi, S. Fukuzumi and W. Nam, Nat. Chem., 2014, 6, 934-940.

148 M. Capone, D. Narzi, D. Bovi and L. Guidoni, J. Phys. Chem. Lett., 2016, 7, 592-596.

149 S. A. Cook and A. S. Borovik, Acc. Chem. Res., 2015, 48, 24072414.

150 R. Gupta, T. Taguchi, B. Lassalle-Kaiser, E. L. Bominaar, J. Yano, M. P. Hendrich and A. S. Borovik, Proc. Natl. Acad. Sci. U. S. A., 2015, 112, 5319-5324.

151 R. Gupta, T. Taguchi, A. S. Borovik and M. P. Hendrich, Inorg. Chem., 2013, 52, 12568-12575.

152 M. Shoji, H. Isobe and K. Yamaguchi, Chem. Phys. Lett., 2015, 636, 172-179.

153 P. E. M. Siegbahn, ChemPhysChem, 2011, 12, 3274-3280.

154 X. Li and P. E. M. Siegbahn, Phys. Chem. Chem. Phys., 2015, 17, 12168-12174.

155 P. E. M. Siegbahn and R. H. Crabtree, J. Am. Chem. Soc., 1999, 121, 117-127.

156 N. Cox and J. Messinger, Biochim. Biophys. Acta, Bioenerg., 2013, 1827, 1020-1030.

157 H. Nilsson, T. Krupnik, J. Kargul and J. Messinger, Biochim. Biophys. Acta, Bioenerg., 2014, 1837, 1257-1262.

158 W. Hillier and T. Wydrzynski, Phys. Chem. Chem. Phys., 2004, 6, 4882-4889.

159 W. Hillier and T. Wydrzynski, Coord. Chem. Rev., 2008, 252, 306-317.

160 T. Noguchi, Philos. Trans. R. Soc. London, Ser. B, 2008, 363, 1189-1195.

161 H. Suzuki, M. Sugiura and T. Noguchi, Biochemistry, 2008, 47, 11024-11030.

162 H. Bao and R. L. Burnap, Proc. Natl. Acad. Sci. U. S. A., 2015, 112, E6139-E6147.

163 A. Klauss, M. Haumann and H. Dau, J. Phys. Chem. B, 2015, 119, 2677-2689.

164 T. Noguchi, Biochim. Biophys. Acta, Bioenerg., 2015, 1847, 35-45.

165 F. Rappaport, N. Ishida, M. Sugiura and A. Boussac, Energy Environ. Sci., 2011, 4, 2520-2524.

166 L. Gerencsér and H. Dau, Biochemistry, 2010, 49, 1009810106.

167 H. Nilsson, F. Rappaport, A. Boussac and J. Messinger, Nat. Commun., 2014, 5, 4305. 